\title{
Preparation and characterization of a novel biological nanosilver fluoride synthesized using green tea extract
}

\author{
Z. Al-Nerabieah, ${ }^{1,{ }^{*}}$ E.A. Arrag ${ }^{1}$ and A. Rajab ${ }^{2}$ \\ ${ }^{1}$ Paediatric Dentistry Department, Dental College, University of Damascus, Syria \\ ${ }^{2}$ Organic Chemistry Department, Faculty of Pharmacy, Syrian Private University (SPU), \\ Damascus, Syria
}

\begin{abstract}
Green tea extract was used to synthesize silver nanoparticles (AgNPs); sodium fluoride was added at the end of the synthesis. Change of colour confirmed the reduction of the silver ions; the formation of AgNPs was confirmed by the appearance of a broad absorption peak at $420 \mathrm{~nm}$. Scanning electron microscopy (SEM) showed that the AgNPs were spherical and their average size was corroborated by dynamic light scattering (DLS) as $8 \mathrm{~nm}$; their zeta potential indicated high stability. The advantage of this "green" method of synthesis of nanosilver fluoride for applications in the dental field, especially in paediatric dentistry, is the absence of hazardous chemicals.
\end{abstract}

Keywords: biological, denistry, green tea extract, nanosilver fluoride

\section{Introduction}

A major part of nanotechnology is concerned with the synthesis of nanoparticles. ${ }^{1}$ Metal nanoparticles, with sizes ranging from 1 to $100 \mathrm{~nm}$, have been found to be useful in many fields due to their unique physicochemical characteristics. ${ }^{2}$ Silver nanoparticles (AgNPs) have drawn considerable attention in biomedicine and dentistry due to their antibacterial properties. ${ }^{3}$ There are three basic methods for the synthesis of AgNPs: chemical, physical and biological.

* Corresponding author. E-mail: Zuhairmajid@gmail.com

1 S. Bayda, M. Adeel, T. Tuccinardi, M. Cordani, F. Rizzolio, The history of nanoscience and nanotechnology: From chemical-physical applications to nanomedicine. Molecules 25 (2020) 112.

2 Y.N. Slavin, J. Asnis, U.O. Häfeli, H. Bach, Metal nanoparticles: understanding the mechanisms behind antibacterial activity. J. Nanobiotechnol. 15 (2017) 65.

3 I.X. Yin, J. Zhang, I.S. Zhao, M.L. Mei, Q. Li, C.H. Chu, The antibacterial mechanism of silver nanoparticles and their application in dentistry. Intl J. Nanomed. 15 (2020) 2555. 
Traditional chemical and physical approaches are extravagant and require complicated laboratory apparatus, ${ }^{4}$ however, which provides the motivation for seeking more efficient methods based on sustainably procurable raw materials.

As a precaution during the current Covid-19 pandemic, dental practitioners and hygienists are mainly providing nonaerosol treatments wherever possible, e.g. the application of fluoride varnishes. In 2014, dos Santos et al. reported that a chemically prepared nanosilver fluoride varnish was able to arrest carious lesions in children's teeth. ${ }^{5}$ Sodium borohydride, a hazardous chemical, ${ }^{6}$ was used in the synthesis. In the last decade, biological reagents have been extensively researched for synthesizing silver nanoparticles with controlled structure. ${ }^{7}$ Some plant leaf extracts can act as reducing agent and stabilizer. These "biological" or "green" methods of synthesis are, furthermore, rapid, simple and low-cost. ${ }^{8}$

Green tea (Camellia sinensis) is one of the most-consumed beverages in Asian countries. There are many favourable effects of consuming green tea as it contains health-promoting bioactive components that may inter alia help prevent cancer and cardiovascular diseases., ${ }^{9,10}$ Prominent among these components are polyphenols, which have been thoroughly studied for use in the synthesis of nanoparticles. The major active components of green tea are catechins and other flavonoids, which can act as reducing and capping agents in the synthesis of AgNPs. ${ }^{11}$

Our interest, however, is in stomatological "silver fluoride" rather than pure elemental silver. Here we propose a novel biological approach to rapidly synthesize nanosilver fluoride (NSF) and investigate its characteristics.

\section{Experimental}

\subsection{Materials}

Silver nitrate $\left(\mathrm{AgNO}_{3}\right)$, potassium carbonate $\left(\mathrm{K}_{2} \mathrm{CO}_{3}\right)$ and sodium fluoride $(\mathrm{NaF})$ were obtained from Sigma-Aldrich. Green tea leaves were obtained from a local market. Ultrapure water was prepared using an Elix Advantage water purification system (Merck, Darmstadt, Germany).

4 P. Singh, Y.-J. Kim, D. Zhang, D.-C. Yang, Biological synthesis of nanoparticles from plants and microorganisms. Trends Biotechnol. 34 (2016) 588-599.

5 V.E. dos Santos Jr, A. Vasconcelos Filho, A.G.R. Targino, M.A.P. Flores, A. Galembeck, A.F. Caldas $\mathrm{Jr}$, A. Rosenblatt, A new "silver bullet" to treat caries in children - nano silver fluoride: a randomised clinical trial. J. Dent. 42 (2014) 945-951.

6 J. Burns, K. Hollands, Nano silver fluoride for preventing caries. Evidence Based Dent. 16 (2015) 8-9.

7 H.D. Beyene, A.A. Werkneh, H.K. Bezabh, T.G. Ambaye, Synthesis paradigm and applications of silver nanoparticles (AgNPs), a review. Sustainable Mater. Technol. 13 (2017) 18-23.

8 S. Rajeshkumar, L. V Bharath, Mechanism of plant-mediated synthesis of silver nanoparticles-a review on biomolecules involved, characterisation and antibacterial activity. Chem. Biol. Interactions 273 (2017) 219-227.

9 L. Xing, H. Zhang, R. Qi, R. Tsao, Y. Mine, Recent advances in the understanding of the health benefits and molecular mechanisms associated with green tea polyphenols, J. Agric. Food Chem. 67 (2019) 1029-1043.

10 W.C. Reygaert, An update on the health benefits of green tea. Beverages 3 (2017) 6.

11 D.A. Selvan, D. Mahendiran, R.S. Kumar, A.K. Rahiman, Garlic, green tea and turmeric extractsmediated green synthesis of silver nanoparticles: Phytochemical, antioxidant and in vitro cytotoxicity studies. J. Photochem. Photobiol. B 180 (2018) 243-252. 


\subsection{Methodology}

\subsubsection{Green tea extract preparation}

3 g green tea leaves were put in $200 \mathrm{~mL}$ distilled water and was heated at $80^{\circ} \mathrm{C}$ for $20 \mathrm{~min}$. The extract was left at room temperature to cool and then filtered through Whatman filter paper №1. Filtered green tea extract was used to synthesis AgNPs.

\subsubsection{Nanosilver fluoride synthesis}

An aliquot of $30 \mathrm{~mL}$ green tea extract was diluted into $250 \mathrm{~mL}$ ultrapure water and stirred until a homogenous solution was obtained. Its $\mathrm{pH}$ was 6 as measured by a pH 211 meter (Hanna Instruments, Padua, Italy). Potassium carbonate $\mathrm{K}_{2} \mathrm{CO}_{3}$ was added until $\mathrm{pH} 10$ was reached (Fig. 1). $20 \mathrm{~mL}$ of $\mathrm{AgNO}_{3}(1 \mathrm{mM})$ was added to the mixture in a single shot and any changes in colour were noted. Finally, a solution of $\mathrm{NaF}(0.526 \mathrm{M})$ was added. The resulting nanosilver fluoride was kept in a dark bottle until further experiments. The final concentrations of each ingredient were: green tea extract [30 $000 \mathrm{mg} / \mathrm{mL}]$; AgNPs [400 mg/mL] and NaF [5000 $\mathrm{mg} / \mathrm{mL}]$.

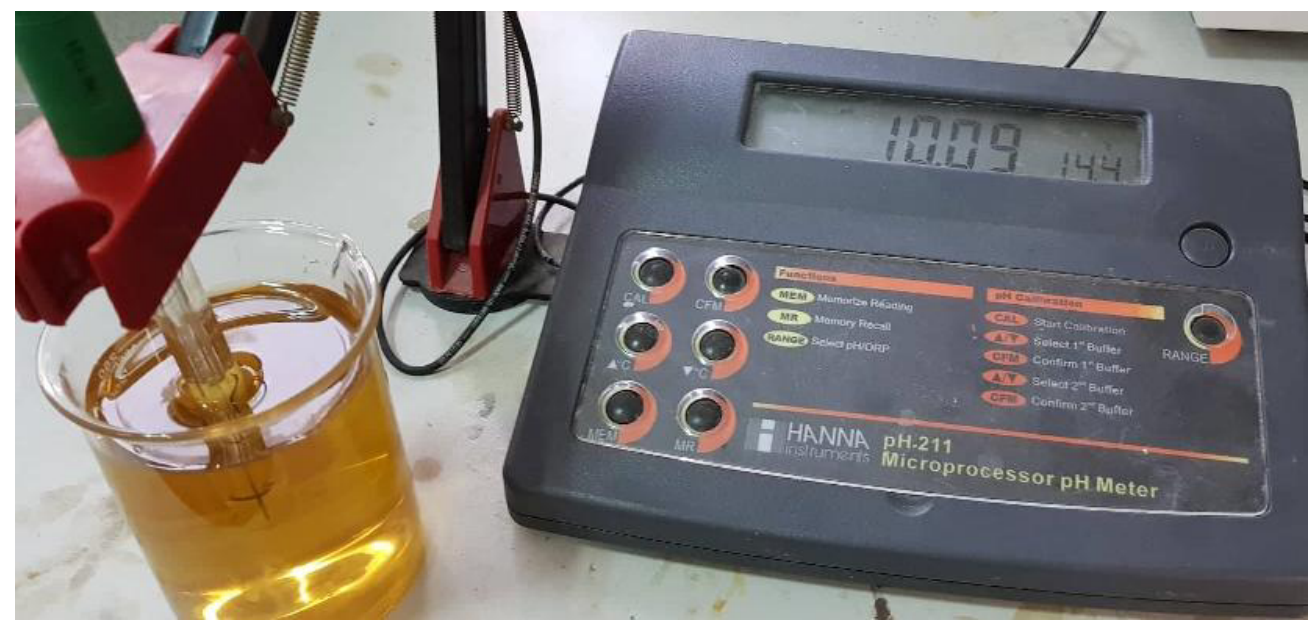

Figure 1. Buffering green tea extract with $\mathrm{K}_{2} \mathrm{CO}_{3}$ until $\mathrm{pH} 10$ was reached.

\subsubsection{Nanosilver fluoride characterization}

\section{Colour change and UV-vis spectroscopy}

After adding the stock solution of $\mathrm{AgNO}_{3}$ to the green tea extract any changes of the mixture's colour were recorded. UV-vis spectroscopy was used to evidence the reduction of $\mathrm{Ag}^{+}$and the formation of nanoparticles. The nanosilver fluoride solution was placed in a quartz cuvette and analysed from 200 to $900 \mathrm{~nm}$ using a Lambda 35 instrument (Perkin Elmer, Long Island, USA) at room temperature. Ultrapure water was used as a reference. 
Scanning electron microscopy (SEM)

A VEGA II scanning electron microscope (TESCAN, Brünn, Czech Republic) was used to determine the morphology of the synthesized nanosilver fluoride. The solution was first centrifuged at 14000 revolutions per minute for $4 \mathrm{~min}$, repeated 3 times with washing between. Then, thin films made by letting a few drops of the supernatant dry were sputtered with gold prior to examination in the ultramicroscope.

\section{Dynamic light scattering (DLS)}

Size and stability of the nanosilver particles were analysed using a Zetasizer Nano ZS90 (Malvern Panalytical, UK). Particle size was measured using DLS at a scattering angle of $90^{\circ}$ at $25^{\circ} \mathrm{C}$ in a disposable cell. The Stokes-Einstein relationship was assumed in order to calculate size and size distribution from the particle diffusivities. The polydispersity index is dimensionless and scaled such that values smaller than 0.05 are rarely seen other than with highly monodisperse standards. Values greater than 0.7 indicate that the sample has a very broad size distribution and is probably not suitable for the DLS technique. The various size distribution algorithms work with data that falls between these two extremes. The calculations for these parameters are defined in ISO standard 22412:2017.

\section{Results - characterization of nanosilver fluoride}

After the addition of $\mathrm{AgNO}_{3}$ to the green tea extract, the light brown colour slowly turned dark, indicating the reduction of $\mathrm{Ag}^{+}$as shown in Fig. 2. The UV-vis spectrum showed a broad absorption peak at $420 \mathrm{~nm}$ (Fig. 3). SEM showed that the AgNPs were mostly spherical in shape and evidently they were not aggregated. According to the DLS, the average size of the AgNPs was $8 \mathrm{~nm}$, and the polydispersity index was 0.297 . The $\zeta$-potential was $-0.39 \mathrm{mV}$.

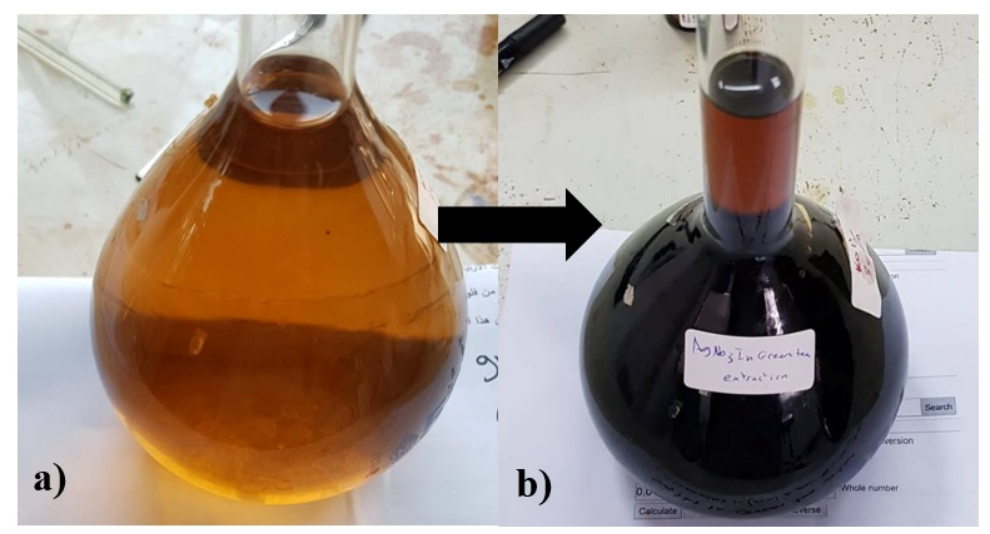

Figure 2. Colour change of green tea extract a), and b) after adding $\mathrm{AgNO}_{3}$. 


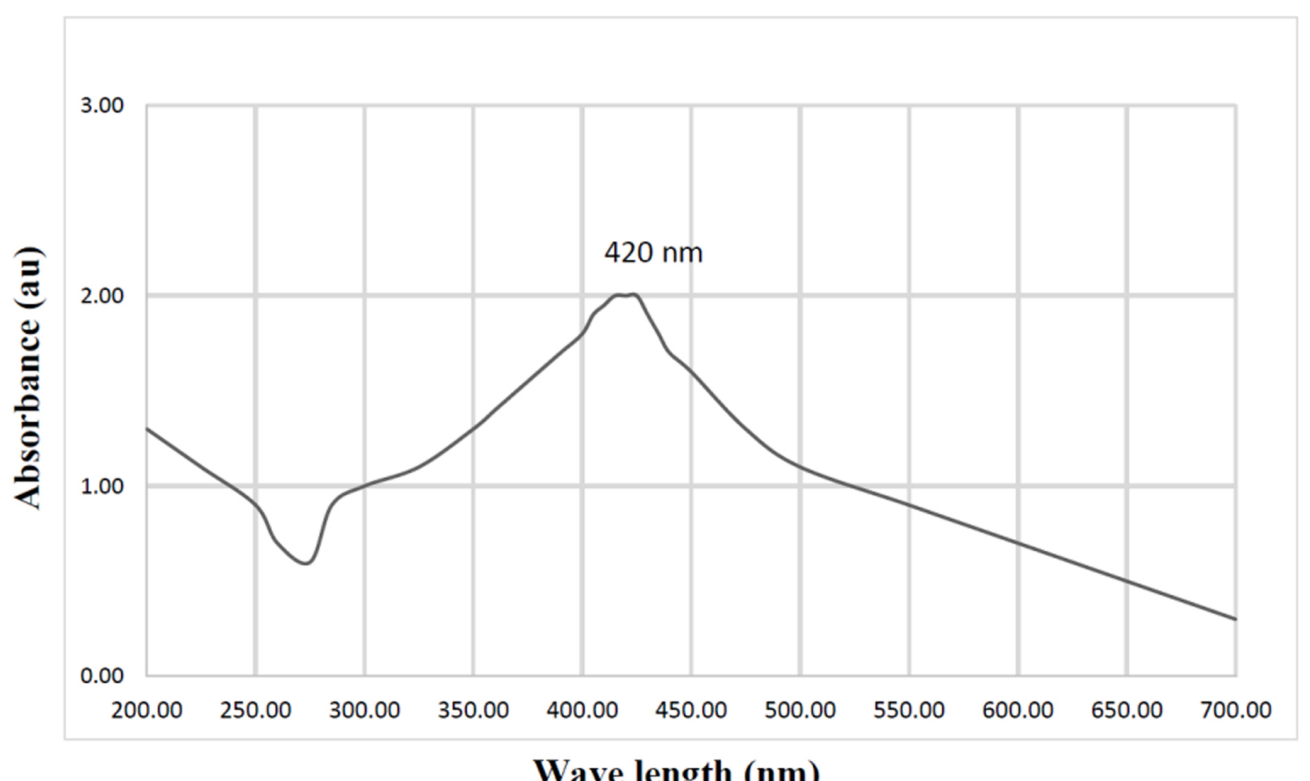

Figure 3. UV-vis spectrum of nanosilver fluoride with green tea extract (corresponding to b) in Fig. 2).

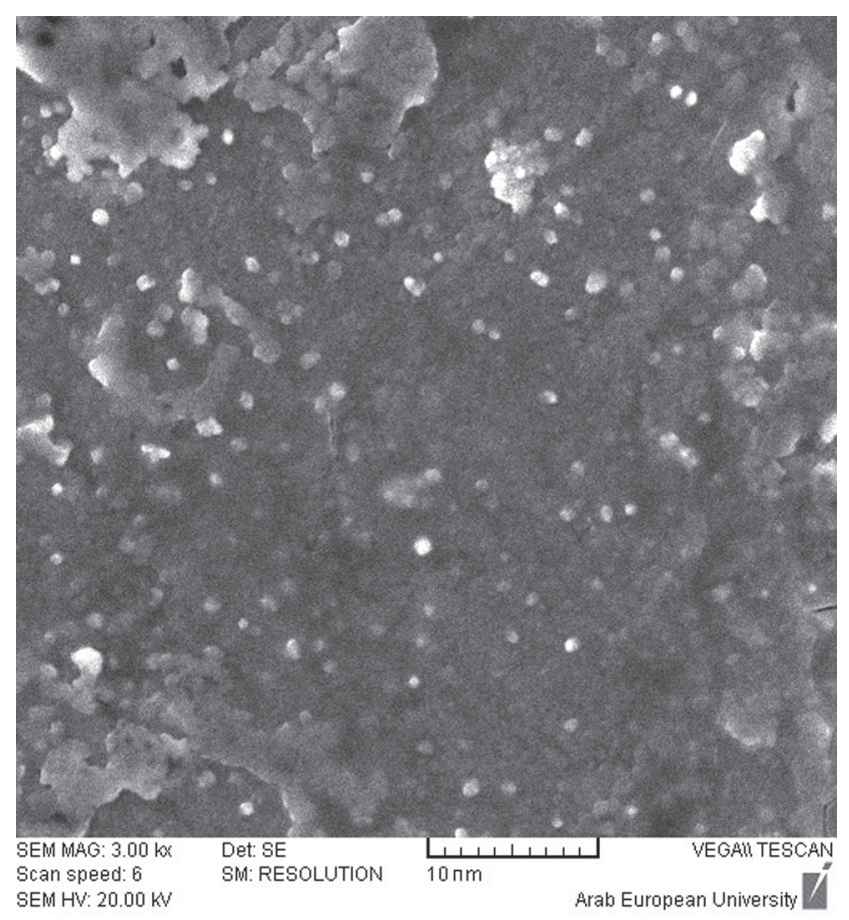

N-ALkafri

Figure 4. Scanning electron micrographs of the final preparation of nanosilver fluoride. 


\section{Discussion}

Buffering green tea extract with alkali was adapted from previous studies reporting that it produced controlled and stabilized AgNPs. ${ }^{12,13} \mathrm{NaF}$ was added as a final step to improve the stability, antibacterial potency and dental remineralization efficacy of the preparation. ${ }^{14}$

The change of colour upon adding $\mathrm{AgNO}_{3}$ is an indication of $\mathrm{Ag}^{+}$reduction by the green tea extract. Other studies have reported the same phenomenon when using green tea extract to synthesis AgNPs. It is attributed to the excitation of surface plasmon resonance, ${ }^{15-17}$ which engenders the broad absorption peak at $420 \mathrm{~nm}$ (Fig. 3). It is in agreement with earlier findings of peak absorption of silver nanoparticles ranging from 415 to $430 \mathrm{~nm} .7,18$

SEM revealed that the particles were spherical in shape and not aggregated. This observation was in accordance with the UV-vis spectrum; the single absorption peak is consistent with spherical AgNPs (while two or more peaks would imply irregular shapes).

The DLS result of an average size of the AgNPs of $8 \mathrm{~nm}$ is in agreement with the scanning electron micrographs, which similarly corroborate the narrow size distribution inferred from the polydispersity index.

The relatively high $\zeta$-potential implies that the colloid is stable with respect to flocculation, ${ }^{19}$ and indeed our preparation was found to be stable. Green tea components are considered to act as a stabilizers of AgNPs, preventing undesired aggregation, ${ }^{13,15,20}$ albeit that the flavonoid components are not ions, ${ }^{21,22}$ hence the origin of the negative $\zeta$-potential may have to be sought elsewhere. It has been reported that $\mathrm{NaF}$ increases the stability of AgNPs, ${ }^{14}$ presumably by the adsorption of fluoride ions to the silver. In effect we have a composite structure with a silver core and a thin shell of silver fluoride.

12 F. Cataldo, Green synthesis of silver nanoparticles by the action of black or green tea infusions on silver ions. Eur. Chem. Bull. 3 (2014) 280-289.

13 W.F. Elbossaty, Green tea as biological system for the synthesis of silver nanoparticles. J. Biotechnol. Biomater. 7 (2017) 3-7.

14 A.G.R. Targino, M.A.P. Flores, V.E. dos Santos Junior, F. de G.B. Bezerra, H. de Luna Freire, A. Galembeck, A. Rosenblatt, An innovative approach to treating dental decay in children. A new anti-caries agent. J. Mater. Sci. Mater. Med. 25 (2014) 2041-2047.

15 A. Chandra, A. Bhattarai, A.K. Yadav, J. Adhikari, M. Singh, B. Giri, Green synthesis of silver nanoparticles using tea leaves from three different elevations. ChemistrySelect 5 (2020) 4239-4246.

16 W.R. Rolim, M.T. Pelegrino, B. de Araújo Lima, L.S. Ferraz, F.N. Costa, J.S. Bernardes, T. Rodigues, M. Brocchi, A.B. Seabra, Green tea extract mediated biogenic synthesis of silver nanoparticles: Characterization, cytotoxicity evaluation and antibacterial activity. Appl. Surf. Sci. 463 (2019) 66-74.

17 I. Al-Ogaidi, M.I. Salman, F.I. Mohammad, Z. Aguilar, M. Al-Ogaidi, Y.A. Hadi, R.M. Al-Rhman, Antibacterial and cytotoxicity of silver nanoparticles synthesized in green and black tea. World J. Exp. Biosci. 5 13Q (2017) 39-45.

18 L. Ge, Q. Li, M. Wang, J. Ouyang, X. Li, M.M.Q. Xing, Nanosilver particles in medical applications: synthesis, performance, and toxicity. Intl J. Nanomed. 9 (2014) 2399.

19 J.D. Clogston, A.K. Patri, Zeta potential measurement. Methods Molec. Biol. 697 (2011) 63-70.

20 W.R. Rolim, M.T. Pelegrino, B. de Araújo Lima, L.S. Ferraz, F.N. Costa, J.S. Bernardes, T. Rodigues, M. Brocchi, A.B. Seabra, Green tea extract mediated biogenic synthesis of silver nanoparticles: Characterization, cytotoxicity evaluation and antibacterial activity. Appl. Surf. Sci. 463 (2019) 66-74.

21 R.A. Riemersma, C.A. Rice-Evans, R.M. Tyrrell, M.N. Clifford, M.E.J. Lean, Tea flavonoids and cardiovascular health. Q. J. Med. 94 (2001) 277-282.

22 G.J. Du, Z. Zhang, X.D. Wen, C. Yu, T. Calway, C.S. Yuan, C.Z. Wang. Epigallocatechin gallate (EGCG) is the most effective cancer chemopreventive polyphenol in green tea. Nutrients 4 (2012) 1679-1691. 
It was reported recently that the degree of antibacterial efficacy of AgNPs depends on the shape and size of the particles. Small spherical ones demonstrated higher antibacterial efficacy than others. ${ }^{23}$ In vitro studies found that AgNPs synthesized using green tea extract gives high antibacterial efficacy. ${ }^{\mathbf{1 6}}$ In vivo studies are required to assess the cariostatic efficacy of the novel nanosilver fluoride described here.

\section{Conclusions}

In this study, an approach using biological ingredients instead of a chemical method was proposed to synthesize nanosilver fluoride. The synthesis was simple, rapid, cost-effective and avoided the use of hazardous reagents. This novel preparation may have beneficial uses as a varnish in the dental field, especially in paediatric dentistry.

\section{Acknowledgments}

The authors are grateful to the Atomic Energy Commission of Syria (AECS) for experimental support. They thank Dr Safaa Shehabi for her assistance with the SEM measurements.

${ }^{23}$ M.A. Raza, Z. Kanwal, A. Rauf, A.N. Sabri, S. Riaz, S. Naseem, Size-and shape-dependent antibacterial studies of silver nanoparticles synthesized by wet chemical routes. Nanomaterials $\mathbf{6}$ (2016) 74 . 